\title{
Caractérisation et stratification d'une collection de mil (Pennisetum glaucum (L.) R. Br.) du Burkina Faso
}

\author{
Roger G. ZANGRE ${ }^{1}$, Mahamadou SAWADOGO ${ }^{2 *}$, Mahamadi OUEDRAOGO ${ }^{3}$ et \\ Didier BALMA ${ }^{4}$
}

${ }^{1}$ Agence Nationale de Valorisation des Acquis de la Recherche (ANVAR/CNRST) - Ouagadougou, 01 BP 476 Ouagadougou 01, Burkina Faso.

${ }^{2}$ Université de Ouagadougou/Unité de Formation et de Recherche en Science de la vie et de la Terre

${ }^{3}$ Institut de l'Environnement et de Recherches Agricoles (INERA/CREAF Kamboinsé) Ouagadougou, O1 BP 476 Ouagadougou 01, Burkina Faso.

${ }^{4}$ Direction de la Recherche Scientifique (DRS/DGESRS) - Ouagadougou, Burkina Faso.

*Auteur correspondant, (UO-UFR/SVT)-Ouagadougou, 9 BP 848 Ouagadougou 09, Burkina Faso.

Tél : +226 70234587, E-mail : sawadogomahamadou@yahoo.fr, sawma@univ-ouaga.bf

\section{RESUME}

Soixante dix (70) écotypes de mil (Pennisetum glaucum (L.) R. Br.) collectés avec la participation des paysans dans huit régions du Burkina Faso, ont été évalués sur le plan agro-morphologique en vue de comprendre la nomination des variétés par le paysan, de décrire et de stratifier la variabilité à des fins de conservation et d'utilisation dans les programmes de sélection. L'étude a montré que les noms utilisés par les paysans pour désigner les écotypes varient d'une région á l'autre et sont toujours en relation avec des caractéristiques agromorphologiques ou avec le cycle végétatif. Elle a montré que pour certains paramètres tels que le poids moyen de l'épi, le nombre d'épis récoltés, le nombre moyen d'épis par plante, la longueur de l'épi et le rendement en grains, les coefficients de variation $(\mathrm{CV})$ sont très élevés (>20\%) au niveau de l'ensemble de la collection mais moins $(<10)$ á l'intérieur de chaque région. Par ailleurs, la structuration de la population sur la base du cycle et des caractères morphologiques y compris le rendement, engendre systématiquement une structuration régionale regroupant d'une part les écotypes du nord, du centre-nord et de l'ouest (groupe 1) et d'autre part ceux du sud, du centre-sud et du centre-est (groupe 2). La Classification Ascendante Hiérarchique $(\mathrm{CAH})$ a aussi permis de dégager 13 classes homogènes dont 8 du groupe 1 et 5 du groupe 2 montrant $88 \%$ de variation interclasse.

() 2009 International Formulae Group. All rights reserved.

Mots clés: Ecotype, variabilité agromorphologique, diversité génétique.

\section{INTRODUCTION}

Le mil, céréale d'origine africaine se cultive dans les zones les plus diverses de l'Afrique. La diversité génétique du mil trouvée en Afrique ne laisse aucun doute que le mil provient des zones sahéliennes de l'Afrique (Obilama et Manyasa, 2002). Cette diversité de zones agroécologiques confère au mil une importante diversité génétique constatée pour cette céréale. L'importance de la variabilité des mils se traduit ça et là par une multiplicité de noms locaux (Beninga, 1992). Au Burkina Faso, des prospections et des collectes ont permis de réunir un nombre important d'échantillons conservés dans des banques de gènes ou exploités dans les programmes de sélection. Les problèmes liés au nombre important des échantillons à conserver ont amené des auteurs à proposer des stratégies de limitation du nombre d'échantillons á conserver par la constitution de "core collection" (Frankel and Brown, 
1984) d'une part et par la réduction du nombre d'échantillons collectés par l'approche participative d'autre part (Christinck et al., 2000). L'approche participative impliquant les obtenteurs et les producteurs à tous les niveaux de la sélection est aussi un outil efficace pour la constitution des "core collection" (Marcos et al., 2000). La collecte par la méthode participative permet de réduire le nombre d'échantillons à collecter sans porter atteinte à la diversité devant être collectée. Cependant pour une meilleure utilisation de la collection, il est nécessaire d'évaluer et de caractériser le germplasm. Aussi, durant les campagnes agricoles 2000 et 2001, des collectes d'écotypes locaux dans les régions Nord, Est, Ouest et Sud du Burkina Faso avec la participation des paysans, ont permis de réunir un important lot d'écotypes locaux. Les enquêtes préliminaires réalisées lors de la collecte ont abouti à une connaissance générale du matériel selon la perception des paysans. Cependant cette connaissance générale basée sur des enquêtes ne donne pas une idée précise et objective de la diversité de la collection. Une évaluation agromorphologique s'avère donc nécessaire afin de décrire et de stratifier cette diversité pour une utilisation efficiente et une meilleure conservation de la diversité génétique. Les analyses multivariées telles que l'analyse en composantes principales sont bien indiquées pour l'étude et la stratification de la variabilité chez le mil. Elle pourrait aider á l'identification de régions présentant un maximum de diversité génétique et de grouper les accessions pour une utilisation effective et la conservation (Pernes et al., 1984). Le présent travail qui se veut être une étape préliminaire à la constitution d'une core collection des mils du Burkina Faso se fixe pour objectifs de décrire et de stratifier la variabilité agromorphologique de la collection en vue d'une meilleure utilisation et une meilleure conservation de la diversité génétique de l'espèce.

\section{MATERIEL ET MÉTHODES Matériel}

Le matériel est composé de soixante dix (70) écotypes de mil collectés dans huit régions différentes du Burkina Faso que sont le Nord (Yatenga), le Nord-Ouest
(Dédougou), l'Ouest (Bobo-Dioulasso), le Centre-Sud (Manga), le Centre-Est (Tenkodogo), le Sud (Zabré), le Centre-Nord (Tougouri), et le Sahel (Pobé-Mengao).

\section{Méthodes}

Les essais de caractérisation ont été installés à Gampéla (station de recherche universitaire située à $15 \mathrm{Km}$ de Ouagadougou) suivant un dispositif de bloc randomisé à 3 répétitions. Chaque entrée est semée sur une ligne à douze (12) poquets aux écartements de $0,8 \mathrm{~cm} \times 0,6 \mathrm{~cm}$, les 10 poquets centraux représentant la parcelle utile.

Deux sarclo-binages et un buttage ont constitué les opérations d'entretien. Un premier apport d'engrais minéral du complexe NPK a été appliqué en couverture à la dose de $100 \mathrm{~kg} / \mathrm{ha}$ et un autre en engrais azoté sous forme d'Urée à $46 \% \mathrm{~N}$ à la dose de $50 \mathrm{~kg} / \mathrm{ha}$, a été réalisé à la montaison.

Un ensemble de dix huit (20) variables y compris les composantes du rendement a été observé et calculé selon Zangré et al. (1992). Il s'agit :

- des caractères $50 \%$ Floraison $(50 \%$ F), nombre de pieds récoltés (NPR), nombre d'épis récoltés (NER), poids des épis récoltés (PER) et poids grains des épis récoltés (PGER) mesurés sur l'ensemble des 10 plantes centrales ;

- des caractères hauteur de la plante à maturité (HMP), nombre de talles basales productives (NTBP), longueur de l'épi (LOE), largeur de l'épi (LAE), diamètre de la tige au niveau de l'entre-nœud 3-4 (D34), longueur et largeur du pédoncule (LOP et LAP), longueur et largeur du drapeau (LOD et LAD) mesurés sur 5 plantes aléatoires de la parcelle utile ;

- de six (6) variables calculées comprenant le poids moyen de l'épi (PME), le poids moyen des grains par épi (PMGE), le nombre d'épis récoltés par plante (NERP), le rendement total épi (RDTE), le rendement total grains (RDTG) et le rapport grains sur épi (G/E).

Les données ont fait l'objet :

- d'une analyse de variance simple réalisée avec l'option "One-way ANOVA" du logiciel SAS et de STAT-ITCF. Les liaisons entre variables ont été mises en évidence par le calcul des corrélations totales. 
- d'une analyse multivariée prenant en compte les variables les plus discriminantes. Il a donc été possible avec le logiciel STATITCF d'effectuer une stratification de l'ensemble de la collection en groupes d'individus homogènes grâce à l'analyse en composantes principales (ACP) et à la classification ascendante hiérarchique $(\mathrm{CAH})$ avec troncature automatique de l'arbre hiérarchique. L'ACP est effectuée sur des données centrées-réduites et les matrices de corrélation, prenant en compte les variables les plus discriminantes. Les contributions relatives des axes et les variables explicatives ont servi au choix des axes à retenir.

Suite à l'ACP, les coordonnées des individus sur les composantes retenues ont été conservées pour servir de données à une $\mathrm{CAH}$ $\mathrm{du}$ moment d'ordre deux. Les groupes d'individus semblables ont été constitués grâce à la troncature automatique de l'arbre hiérarchique en maximisant la variance interclasse. Le bien fondé de ces groupes est ensuite testé par l'analyse factorielle discriminante (AFD) et l'analyse de variance en utilisant les données brutes.

\section{RÉSULTATS}

Variabilité des caractères de la collection

Les résultats présentés dans le tableau 1 montrent que hors mis quelques variables tels que NTBP, LAD, NPR, RDTE, RDTG et G/E, la majorité présente une moyenne hautement significative au seuil de 0,01. L'échantillon étudié présente des écotypes de grandes tailles ayant une floraison très étalée dans le temps (51 jours pour les plus précoces et 77 jours pour les plus tardifs). Ceci donne, en supposant la période de maturation du mil de 30 à 35 jours après floraison, des cycles de 80 - 85 jours et de $107-112$ jours. Ce qui correspondrait à des écotypes de cycle précoce et moyen; même si ces plantes possèdent des épis de taille très variable $(26-$ $67 \mathrm{~cm}$ pour la longueur et $1,6-2,4 \mathrm{~cm}$ pour la largeur), le tallage et le nombre d'épis récoltés sont faibles (3 à 6 talles productives par plante et 18 à 48 épis récoltés par parcelle de 10 poquets). L'on observe également une grande variabilité pour les variables composant le rendement tels que le poids moyen de l'épi et des grains, le nombre d'épis récoltés, le nombre moyen d'épis par plante, la longueur de l'épi et le rendement grain avec un coefficient de variation (C.V) supérieur à vingt pour cent (> 20\%).

La variabilité se traduit aussi par le nombre de classes distinctes obtenues par le test de comparaison des moyennes (test de Newman-Keuls au seuil de 1\%). Il faut noter que les caractères hauteur de la plante, longueur de l'épi, largeur du drapeau et longueur du pédoncule présentent une distribution plurimodale traduisant l'existence possible de sous-populations.

\section{Associations des caractères}

Les relations entre les variables sont décrites par les coefficients de corrélations totales présentées dans le tableau 2. Le cycle $50 \%$ floraison est fortement et positivement corrélé à la hauteur de la plante, à la longueur du drapeau et à la longueur du pédoncule. La longueur de l'épi est liée au diamètre de la tige, au diamètre du pédoncule et au poids moyen de l'épi. La largeur de l'épi se trouve négativement corrélée à la longueur du pédoncule. Le rendement est corrélé au nombre d'épis récoltés, au rapport grain sur épi, et au nombre d'épis moyen par plante.

\section{Caractérisation des sous-populations et variation des caractères}

La variation des caractères à l'intérieur des régions reste dans l'ensemble inférieure à celle constatée dans l'ensemble de la collection (Tableau 3). Toutefois des C.V de plus $30 \%$ ont été observés pour les variables poids moyen des épis et poids en grains pour la région du sud. Les variations sont faibles pour toutes les régions en ce qui concerne les caractères $50 \%$ floraison, hauteur de la plante à maturité, largeur de l'épi, tandis que les autres variables montrent des C.V variant énormément d'une région à l'autre. Cependant les écarts d'effectifs entre les localités rendent difficiles les comparaisons. D'une manière générale, le nombre d'épis récoltés, le rendement en grains et le poids moyen en grains montrent des C.V élevés dans toutes les régions. Les écotypes du sud, du centre-sud et de l'Ouest se révèlent être les plus tardifs $(50 \% \mathrm{~F}>65$ jours $)$; les plus précoces étant celles du nord, du centre-nord et du sahel. Les écotypes tardifs sont celles ayant donné 
Tableau 1: Paramètres descriptifs et d'analyse de variance de la population.

\begin{tabular}{|c|c|c|c|c|c|c|c|c|c|c|c|c|c|c|c|c|c|c|}
\hline & NER & NTBP & HPM & LAE & LOE & D34 & LOP & LOD & LAD & DAP & NPR & RDTE & RDTG & G/E & $50 \% \mathrm{~F}$ & PME & PGER & NERP \\
\hline Minimum & 18 & 3 & 185 & 16 & 26 & 9 & 35 & 28 & 2 & 5 & 8 & 905 & 488 & 37 & 51 & 16 & 9 & 2 \\
\hline Maximum & 47 & 6 & 275 & 24 & 67 & 14 & 50 & 49 & 3 & 9 & 10 & 2671 & 1588 & 71 & 77 & 57 & 33 & 5 \\
\hline Moyenne & 33 & 4 & 225 & 20 & 35 & 11 & 42 & 36 & 3 & 7 & 10 & 1723 & 1007 & 58 & 65 & 26 & 15 & 3 \\
\hline E T & 6.8 & 0.7 & 23.4 & 1.9 & 7.2 & 0.9 & 4.2 & 4.3 & 0.3 & 0.7 & 0.4 & 343.0 & 234.1 & 6.4 & 5.5 & 6.7 & 3.8 & 0.7 \\
\hline C.V & 21.0 & 15.5 & 10.4 & 9.4 & 20.4 & 8.3 & 10.0 & 11.9 & 10.5 & 10.2 & 4.0 & 19.9 & 23.2 & 11.0 & 8.5 & 25.4 & 24.4 & 20.8 \\
\hline $\mathrm{F}^{*} 1$ & $S$ & NS & $S$ & $S$ & $\mathrm{~S}$ & $\mathrm{~S}$ & $\mathrm{~S}$ & $\mathrm{~S}$ & NS & $S$ & NS & NS & NS & NS & $S$ & $S$ & $S$ & $S$ \\
\hline $\mathrm{Nb}$ classes & 1 & 1 & 18 & 11 & 22 & 3 & 7 & 3 & 1 & 9 & 1 & 1 & 1 & 1 & 3 & 5 & 5 & 1 \\
\hline
\end{tabular}


Tableau 2: Corrélations totales entre variables.

\begin{tabular}{|c|c|c|c|c|c|c|c|c|c|c|c|c|c|c|c|c|c|}
\hline & $50 \% \mathrm{~F}$ & HPM & LOE & LAE & LOD & LOP & DAP & D34 & NER & PME & PGER & RDTG & G/E & NTBP & LAD & NPR I & NERP \\
\hline $50 \% \mathrm{~F}$ & 1 & & & & & & & & & & & & & & & & \\
\hline HPM & $0.75 * *$ & 1.00 & & & & & & & & & & & & & & & \\
\hline LOE & -0.11 & -0.01 & 1.00 & & & & & & & & & & & & & & \\
\hline LAE & $-0.21 *$ & $-0.28 * *$ & 0.02 & 1.00 & & & & & & & & & & & & & \\
\hline LOD & $0.53 * *$ & $0.53 * *$ & $0.28 * *$ & -0.08 & 1.00 & & & & & & & & & & & & \\
\hline LOP & $0.65 * *$ & $0.73 * *$ & $-0.33 * *$ & $-0.52 * *$ & $0.40^{* *}$ & 1.00 & & & & & & & & & & & \\
\hline DAP & -0.17 & $-0.21^{*}$ & $0.79 * *$ & $0.31 * *$ & $0.30 * *$ & $-0.44 * *$ & 1.00 & & & & & & & & & & \\
\hline D34 & $0.23 *$ & $0.28 * *$ & $0.61 * *$ & $0.34 * *$ & $0.51 * *$ & -0.08 & $0.70^{* *}$ & 1.00 & & & & & & & & & \\
\hline NER & -0.03 & 0.03 & $-0.43 * *$ & 0.00 & $-0.25^{*}$ & 0.18 & $-0.48 * *$ & $-0.34 * *$ & 1.00 & & & & & & & & \\
\hline PME & -0.17 & -0.08 & $0.52 * *$ & $0.30 * *$ & 0.10 & $-0.37 * *$ & $0.56 * *$ & $0.51 * *$ & $-0.51 * *$ & 1.00 & & & & & & & \\
\hline PGER & $-0.25^{*}$ & -0.10 & $0.32 * *$ & $0.25 *$ & -0.09 & $-0.32 * *$ & $0.32 * *$ & $0.34 * *$ & $-0.38 * *$ & $0.90 * *$ & 1.00 & & & & & & \\
\hline RDTG & $-0.36^{* *}$ & -0.15 & -0.07 & $0.31 * *$ & $-0.37 * *$ & $-0.23 *$ & -0.09 & 0.00 & $0.62 * *$ & 0.17 & $0.40 * *$ & 1.00 & & & & & \\
\hline G/E & -0.20 & -0.07 & $-0.38 * *$ & -0.13 & $-0.46^{* *}$ & 0.08 & $-0.53^{* *}$ & $-0.39 * *$ & $0.35^{* *}$ & $-0.23 *$ & 0.20 & $0.53 * *$ & 1.00 & & & & \\
\hline NTBP & $0.23 *$ & $0.30 * *$ & $-0.44 * *$ & $-0.22 *$ & -0.07 & $0.43^{* *}$ & $-0.54 * *$ & $-0.27 *$ & $0.66^{* *}$ & $-0.49 * *$ & $-0.37 * *$ & $0.25 *$ & $0.31 * *$ & 1.00 & & & \\
\hline LAD & 0.03 & -0.06 & $0.38 * *$ & $0.23 *$ & $0.27 *$ & -0.07 & $0.51 * *$ & $0.37 * *$ & $-0.25^{*}$ & $0.31 * *$ & 0.16 & -0.06 & $-0.41 * *$ & $-0.21 *$ & 1.00 & & \\
\hline NPR & 0.06 & 0.11 & 0.00 & $0.26^{*}$ & 0.04 & 0.02 & 0.11 & 0.07 & 0.13 & 0.06 & 0.02 & 0.15 & -0.07 & -0.06 & $0.22 *$ & 1.00 & \\
\hline NERP & -0.05 & 0.01 & $-0.43 * *$ & -0.05 & $-0.27 *$ & 0.17 & $-0.50 * *$ & $-0.36 * *$ & $0.98 * *$ & $-0.53 * *$ & $-0.38 * *$ & $0.60 * *$ & $0.37 * *$ & $0.68 * *$ & $-0.28 * *$ & -0.07 & 1.00 \\
\hline RDTE & $-0.35^{* *}$ & -0.17 & 0.14 & $0.43^{* *}$ & $-0.22 *$ & $-0.34 * *$ & 0.19 & 0.19 & $0.52 * *$ & $0.36^{* *}$ & $0.38^{* *}$ & $0.87 * *$ & 0.08 & 0.10 & 0.14 & $0.20 *$ & $0.49^{* *}$ \\
\hline
\end{tabular}

NER - nombre d'épis récoltés, NTBP - nombre de talles basales productives, HPM - hauteur de la plante à maturité, LAE - largeur de l'épi, LOE - longueur de l'épi, D34 - diamètre de la tige au niveau de l'entre-nœud 3-4, LOP - longueur du pédoncule, LOD - longueur du drapeau, LAD - largeur du drapeau, DAP - Diamètre du pédoncule, NPR - nombre de pieds récoltés, RDTE - rendement total épi, RDTG - rendement total grains, G/E - rapport grains sur épi, 50\% F - 50\% Floraison, PME - poids moyen de l'épi, PGER - poids grains des épis récoltés et NERP - le nombre d'épis récoltés par plante 
R. G. ZANGRE et al. / Int. J. Biol. Chem. Sci. 3(5): 1042-1056, 2009

Tableau 3: Paramètres descriptifs et d'analyse de variance des sous-populations.

\begin{tabular}{|c|c|c|c|c|c|c|c|c|c|c|c|c|c|c|c|c|c|c|}
\hline Localité & $50 \%$ F & NPD & NER & NTBF & HPM & LOE & LAE & D34 & LOD & LAD & DAP & RDTE & RDTG & GE & PME & PGE & NEP & LOP \\
\hline Bcle Mhn & 63.9 & 9.4 & 32.1 & 4.0 & 216.4 & 40.2 & 19.3 & 11.0 & 34.0 & 2.7 & 7.3 & 1672.4 & 1001.4 & 60.0 & 25.4 & 15.1 & 3.6 & 40.6 \\
\hline C-est & 68.0 & 10.0 & 37.0 & 4.3 & 230.0 & 27.0 & 23.0 & 11.0 & 35.0 & 2.3 & 6.7 & 1725.7 & 1012.0 & 59.1 & 22.3 & 13.1 & 4.0 & 41.3 \\
\hline C-nord & 60.6 & 9.7 & 36.1 & 4.5 & 216.5 & 36.6 & 21.4 & 11.0 & 33.7 & 2.5 & 6.9 & 2091.2 & 1255.7 & 60.0 & 28.6 & 16.9 & 3.8 & 37.6 \\
\hline C-sud & 70.7 & 9.7 & 35.0 & 4.7 & 250.9 & 31.9 & 18.9 & 10.9 & 38.0 & 2.4 & 6.4 & 1631.4 & 1001.8 & 60.8 & 24.3 & 15.0 & 3.7 & 47.0 \\
\hline Nord & 61.0 & 9.7 & 35.3 & 4.0 & 201.3 & 33.3 & 22.7 & 11.3 & 34.0 & 2.3 & 7.0 & 1895.0 & 1166.2 & 61.1 & 25.7 & 15.8 & 4.0 & 37.7 \\
\hline Sahel & 61.5 & 9.9 & 28.4 & 3.9 & 203.7 & 39.8 & 20.5 & 11.3 & 35.5 & 2.9 & 7.7 & 1780.3 & 961.3 & 54.3 & 30.9 & 16.9 & 2.9 & 38.4 \\
\hline Sud & 68.8 & 9.7 & 31.5 & 4.4 & 244.2 & 31.7 & 18.4 & 10.8 & 38.2 & 2.4 & 6.6 & 1435.2 & 840.6 & 58.0 & 22.8 & 13.4 & 3.3 & 45.2 \\
\hline Houet & 77.3 & 9.7 & 29.3 & 3.8 & 248.1 & 38.8 & 22.6 & 12.8 & 48.9 & 2.9 & 8.0 & 1452.8 & 537.5 & 36.9 & 23.9 & 8.9 & 3.0 & 41.2 \\
\hline Minimum & 60.6 & 9.4 & 28.4 & 3.8 & 201.3 & 27.0 & 18.0 & 10.0 & 30.0 & 2.3 & 6.4 & 1435.2 & 537.5 & 36.9 & 22.3 & 8.9 & 2.9 & 37.6 \\
\hline Maximum & 77.3 & 10.0 & 37.0 & 4.7 & 250.9 & 40.2 & 23.0 & 12.8 & 48.9 & 2.9 & 8.0 & 2091.2 & 1255.7 & 61.7 & 30.9 & 16.9 & 4.0 & 47.0 \\
\hline Moyenne & 65.9 & 9.7 & 32.9 & 4.2 & 225.9 & 35.1 & 20.5 & 11.1 & 36.4 & 2.5 & 7.0 & 1710.0 & 979.6 & 56.9 & 25.6 & 14.6 & 3.5 & 41.1 \\
\hline $\mathbf{E} \mathbf{T}$ & 5.4 & 0.2 & 2.9 & 0.3 & 17.5 & 4.2 & 1.8 & 0.7 & 5.0 & 0.2 & 0.5 & 192.6 & 192.0 & 7.4 & 2.6 & 2.4 & 0.4 & 3.0 \\
\hline C.V & 8.2 & 1.7 & 8.8 & 6.6 & 7.7 & 12.0 & 9.0 & 6.2 & 13.7 & 8.4 & 7.4 & 11.3 & 19.6 & 12.9 & 10.2 & 16.4 & 10.5 & 7.4 \\
\hline F*Loc & $\mathrm{S} * *$ & NS & NS & $\mathrm{S} * *$ & $\mathrm{~S} * *$ & $\mathrm{~S} * *$ & $\mathrm{~S} * *$ & NS & $\mathrm{S} * *$ & NS & $\mathrm{S} * *$ & $\mathrm{~S} * *$ & $\mathrm{~S} * *$ & $\mathrm{~S} * *$ & NS & NS & NS & $\mathrm{S} * *$ \\
\hline
\end{tabular}


le moins de talles productives. L'analyse de différences hautement significatives pour les 11 variables suivantes: $50 \%$ floraison, nombre de talles basales productives, hauteur de la plante à maturité, longueur et largeur de l'épi, longueur du drapeau, longueur et largeur du pédoncule, rendement épi et grain et le rapport grain sur épi. Cependant les différences ne sont pas significatives pour les autres variables.

\section{Stratification de la variabilité de la collection}

Les trois premières composantes de l'ACP expliquant $73,8 \%$ de l'information ont été retenues. La première composante expliquant 29,9\% de 1'information est liée aux variables longueur de l'épi, diamètre de la tige et du pédoncule et nombre de talles par plante. L'axe 2 qui explique 27,4\% de l'information est lié aux variables $50 \%$ floraison, longueur $\mathrm{du}$ drapeau et rendement grain. L'axe 3 est lié aux rendements épi et grain. C'est l'axe du rendement et de certaines de ces composantes. Il explique 16,5\% de l'information. C'est sur la base de ces informations que la structuration de la population a été effectuée. Les 3 premières composantes de l'AFD expliquent 93,2\% de l'information. L'axe 1 explique 41,4\% de l'information ; il est lié aux variables longueur de l'épi, diamètre du pédoncule, du drapeau et de la tige et nombre de talles moyen par plante: c'est l'axe des dimensions de l'épi, des largeurs et de la capacité de tallage. L'axe 2 qui explique $30,7 \%$ de l'information est lié aux variables $50 \%$ floraison, hauteur de la plante, longueur $\mathrm{du}$ drapeau et du pédoncule : c'est l'axe du cycle et de l'élongation des plantes. L'axe 3 est expliqué par les variables rendement épi et grain et nombre d'épis récoltés : c'est l'axe du rendement et de la quantité d'épis utiles. Le plan principal formé par les axes 1 et 2 explique $72,1 \%$ de l'information. Les variables représentatives sont par ordre décroissant de qualité de représentation: le cycle $50 \%$ floraison, le diamètre du pédoncule, la longueur du drapeau et du pédoncule, le diamètre de la tige, la longueur de l'épi, la hauteur de la plante et la largeur du drapeau et dans une moindre mesure la largeur de l'épi et le nombre de talles par plante (cosinus carré compris entre 0,955 et 0,559 ). variance entre les localités montre des La quasi totalité des caractères agromorphologiques, exception faite du rendement et du nombre des épis, est représentée sur ce plan. Le plan 1-3 qui explique $62,5 \%$ de l'information est décrite par les variables DAP, NER, LOE, NTBP, RDTE, LAD, D34, RDTG et LAE dont les cosinus carrés varient entre 0,966 et 0,698 . Quant au plan 2-3, il contribue pour 51,6\% à l'explication de l'inertie totale et est expliquée par les variables RDTG, 50\%F, HPM, LOD, RDTE, NER et LOP dont la représentativité varie entre 0,974 et 0,598 .

Au total, 13 classes montrant $88 \%$ de variation interclasse ont été obtenues après la troncature automatique de l'arbre hiérarchique dont les résultats sont présentés dans le tableau 4:

- la classe 1 est constituée de 10 entrées précoces de petite taille, d'épis moyens, de faible tallage et de rendement moyen (1063 $\mathrm{kg} / \mathrm{ha}$ ). Il regroupe les écotypes originaires du sahel, du nord et de la boucle du Mouhoun ;

- la classe 2 constituée de 6 entrées se caractérise par un cycle court, de petite taille, des épis moyennement longs $(38,7 \mathrm{~cm})$, un drapeau et un pédoncule larges, un tallage faible et un rendement moyen faible (1044 $\mathrm{kg} / \mathrm{ha}$ ). Il est composé de 4 écotypes du sahel et de 2 écotypes du centre-nord ;

- la classe 3 comporte 5 individus. Les entrées le constituant se caractérisent par des cycles courts, un tallage moyen, des épis courts, beaucoup d'épis récoltés, un rapport grain sur épi élevé et un bon rendement (1249 kg/ha); - la classe 4 comporte 3 entrées à cycle court, tallage moyen, épis longs, larges et lourds. Le rendement moyen est de $1241 \mathrm{~kg} / \mathrm{ha}$;

- la classe 5 est constituée de 7 entrées précoces à épis moyennement longs et larges et d'un bon rendement ( $1369 \mathrm{~kg} / \mathrm{ha})$;

- la classe 6 comporte 7 entrées précoces, de petite taille, de faible rendement $(800 \mathrm{~kg} / \mathrm{ha})$; - la classe 7 constituée de 8 entrées semitardives de grande taille, à tallage abondant, à épis moyennement longs possède un rendement moyen de $1016 \mathrm{~kg} / \mathrm{ha}$;

- la classe 8 comporte 2 entrées semi-tardives à tallage important. Les épis sont moyennement longs. Le nombre d'épis 
R. G. ZANGRE et al. / Int. J. Biol. Chem. Sci. 3(5): 1042-1056, 2009

Tableau 4: Paramètres descriptifs et d'analyse de variance des classes agromorphologiques.

\begin{tabular}{|c|c|c|c|c|c|c|c|c|c|c|c|c|c|c|c|c|c|c|c|}
\hline Classe & $50 \% \mathrm{~F}$ & HPM & LOE & LAE & LOD & LAD & LOP & DAP & D34 & NTBP & NER & RDTE & RDTG & G/E & PME & PGEP & NERP & NPR & Effectif \\
\hline Cla1 & 62 & 208.1 & 35.7 & 20.1 & 32.8 & 2.5 & 39.3 & 6.9 & 10.6 & 4 & 33 & 1838 & 1063 & 58.3 & 26.9 & 15.7 & 4 & 9 & 10 \\
\hline Cla2 & 62 & 204.0 & 38.7 & 20.2 & 35.9 & 2.8 & 38.0 & 7.6 & 11.1 & 4 & 30 & 1885 & 1044 & 54.5 & 30.5 & 16.7 & 3 & 10 & 6 \\
\hline Cla3 & 61 & 202.7 & 27.3 & 20.5 & 30.0 & 2.3 & 38.3 & 6.0 & 9.8 & 5 & 43 & 1850 & 1249 & 67.4 & 20.2 & 13.7 & 5 & 10 & 3 \\
\hline Cla4 & 62 & 222.9 & 42.3 & 22.0 & 36.3 & 2.9 & 38.9 & 7.7 & 12.0 & 4 & 33 & 2336 & 1241 & 53.7 & 34.4 & 18.6 & 3 & 10 & 3 \\
\hline Cla5 & 60 & 214.3 & 34.6 & 22.1 & 32.2 & 2.6 & 38.1 & 6.9 & 11.0 & 4 & 39 & 2158 & 1369 & 63.7 & 27.0 & 17.3 & 4 & 10 & 7 \\
\hline Cla6 & 62 & 204.1 & 39.1 & 20.0 & 34.8 & 2.6 & 39.3 & 7.5 & 11.2 & 4 & 23 & 1390 & 801 & 57.9 & 28.9 & 16.9 & 2 & 9 & 7 \\
\hline Cla7 & 71 & 255.4 & 31.4 & 20.4 & 39.6 & 2.7 & 46.7 & 6.7 & 11.3 & 5 & 37 & 1763 & 1016 & 56.8 & 23.1 & 13.3 & 4 & 10 & 8 \\
\hline Cla8 & 70 & 248.3 & 33.0 & 18.8 & 38.4 & 2.3 & 46.6 & 6.6 & 10.9 & 5 & 47 & 2175 & 1401 & 64.2 & 22.3 & 14.4 & 5 & 10 & 2 \\
\hline Cla9 & 70 & 241.3 & 54.9 & 21.2 & 45.2 & 2.9 & 39.2 & 8.6 & 13.2 & 4 & 24 & 1635 & 765 & 46.5 & 35.0 & 16.8 & 2 & 10 & 4 \\
\hline Cla10 & 70 & 243.2 & 31.5 & 18.3 & 37.1 & 2.4 & 46.0 & 6.3 & 10.6 & 5 & 35 & 1528 & 913 & 59.8 & 24.1 & 14.5 & 4 & 10 & 8 \\
\hline Cla11 & 76 & 270.1 & 33.3 & 19.2 & 37.6 & 2.5 & 44.8 & 6.9 & 11.9 & 4 & 23 & 1271 & 749 & 56.3 & 26.1 & 15.0 & 2 & 10 & 2 \\
\hline Cla12 & 67 & 239.2 & 30.1 & 18.1 & 37.4 & 2.4 & 46.4 & 6.3 & 10.3 & 4 & 29 & 1213 & 733 & 59.6 & 20.6 & 12.5 & 3 & 10 & 6 \\
\hline Cla13 & 61 & 207.5 & 32.9 & 19.3 & 30.7 & 2.2 & 38.6 & 6.7 & 9.6 & 4 & 32 & 1595 & 919 & 59.6 & 24.5 & 14.7 & 3 & 10 & 3 \\
\hline Minimum & 60.3 & 202.7 & 27.3 & 18.1 & 30.0 & 2.2 & 38.0 & 6.0 & 9.6 & 3.6 & 23.2 & 1213.1 & 733.3 & 46.5 & 20.2 & 12.5 & 2.4 & 9.4 & \\
\hline Maximum & 76.3 & 270.1 & 54.9 & 22.1 & 45.2 & 2.9 & 46.7 & 8.6 & 13.2 & 5.3 & 47.0 & 2336.3 & 1401.4 & 67.4 & 35.0 & 18.6 & 4.7 & 10.0 & \\
\hline Moyenne & 65.6 & 227.8 & 35.8 & 20.0 & 36.0 & 2.6 & 41.6 & 7.0 & 11.0 & 4.3 & 32.9 & 1741.4 & 1020.3 & 58.3 & 26.4 & 15.4 & 3.4 & 9.7 & \\
\hline $\mathbf{E} \mathbf{T}$ & 5.2 & 22.0 & 6.8 & 1.2 & 3.9 & 0.2 & 3.6 & 0.7 & 0.9 & 0.6 & 7.1 & 335.1 & 225.6 & 5.1 & 4.5 & 1.7 & 0.7 & 0.2 & \\
\hline C.V & 7.9 & 9.7 & 18.9 & 6.1 & 10.9 & 8.6 & 8.8 & 9.9 & 8.3 & 13.2 & 21.7 & 19.2 & 22.1 & 8.7 & 17.2 & 11.1 & 21.1 & 1.7 & \\
\hline F*Cla & $\mathrm{S}^{* *}$ & $\mathrm{~S} * *$ & $\mathrm{~S}^{* *}$ & $\mathrm{~S}^{* *}$ & $\mathrm{~S}^{* *}$ & $\mathrm{~S}^{* *}$ & $\mathrm{~S}^{* *}$ & $\mathrm{~S}^{* *}$ & $\mathrm{~S}^{* *}$ & $\mathrm{~S}^{* *}$ & $\mathrm{~S}^{* *}$ & $\mathrm{~S}^{* *}$ & $\mathrm{~S} * *$ & $\mathrm{~S}^{* *}$ & $\mathrm{~S}^{* *}$ & NS & $\mathrm{S}^{* *}$ & NS & \\
\hline
\end{tabular}


récoltés et les rendements sont les meilleurs de tous les groupes $(1400 \mathrm{~kg} / \mathrm{ha})$. Ce groupe est constitué d'un écotype du sud et un du centre-sud ;

- la classe 9 est constituée de 4 entrées semitardives de grande taille, d'épis longs $(55 \mathrm{~cm})$ et lourds mais de tallage est faible. Le rendement en grains est faible $(765 \mathrm{~kg} / \mathrm{ha})$;

- la classe 10 regroupe 8 individus semitardifs de grande taille et de tallage important. Les épis sont moyennement longs et minces et de faible poids. Le nombre d'épis récoltés et le rendement sont faibles $(913 \mathrm{~kg} / \mathrm{ha})$.

- la classe 11 est constituée seulement de deux individus les plus tardifs (76 jrs) et les plus hauts. Le tallage, le nombre d'épis récoltés et le rendement moyen sont faibles $(749 \mathrm{~kg} / \mathrm{ha})$;

- la classe 12 est constituée de 6 entrées moyennement tardives de taille moyenne. Les épis sont de longueur moyenne mais de faible diamètre. Ce groupe a donné le plus faible rendement moyen $(733 \mathrm{~kg} / \mathrm{ha})$;

- la classe 13 regroupe 3 écotypes précoces, de taille réduite et de faible tallage. Le rendement moyen obtenu est de $919 \mathrm{~kg} / \mathrm{ha}$.

Une analyse de variance montre que les classes sont statistiquement différentes (seuil de 1\%) pour tous les caractères analysés à l'exception du nombre de plantes après démariage et le poids grain moyen par épi. Les distances de Mahalanobis entre les classes indiquent que la classe 11 est la plus éloignée de toutes (Tableau 5). Les classes 1 et 2 sont les plus proches, suivies des classes 1 et 5 puis 1 et 6 . Les classes 8 et 11 sont les plus éloignées.

La concordance entre structuration agromorphologique et structuration géographique montre l'importance du facteur régional dans la répartition de la diversité génétique (Tableau 6). Cette étude mérite d'être poussée afin de définir des unités d'aire géographique de la diversité génétique nécessaire pour une meilleure collecte et une meilleure conservation in situ.

\section{DISCUSSION}

Variabilité des caractères

L'analyse de variance a révélé l'existence d'une grande variabilité des caractères agromorphologiques de l'ensemble de la collection. Mais l'épi constitue le caractère le plus discriminant entre les écotypes car probablement beaucoup plus influencé par les critères de sélection du paysan qui reposent essentiellement sur les caractéristiques de l'épi, donc du rendement. En effet au Burkina Faso et dans beaucoup de pays sahéliens, le mil est une culture destinée à la consommation humaine de part l'utilisation de ses graines (Rai et al., 1999).

\section{Association des caractères}

Les corrélations totales entre variables ont montré des types de relations fréquemment rencontrées dans presque toutes les évaluations d'écotypes du mil ; confirmant ainsi la pertinence des caractères étudiés dans la description de la diversité génétique. Néanmoins, il faut retenir que les plantes de grande taille sont celles possédant un cycle tardif.

\section{Relations entre les noms locaux des écotypes et les caractéristiques agromorphologiques}

Les paysans ont des noms locaux pour désigner leurs variétés. On constate que ces noms sont variables selon les régions et font référence dans la plupart des cas à des caractéristiques agromorphologiques ou lié au cycle. En effet la même signification peut revêtir différents niveaux du caractère considéré quand on passe d'une région à l'autre. C'est le cas de la précocité qui correspond à un cycle $50 \%$ floraison de 60 jours pour le Sahel et 70 jours pour la région du Sud. En plus il se pose aussi le problème de limites claires entre les désignations cycle long et cycle précoce au sein de la même région. Ainsi, les résultats laissent apparaître un problème de terminologie. Des investigations plus poussées sont indispensables pour mieux préciser les différentes significations afin qu'elles puissent servir comme outil dans la sélection participative et la gestion des ressources phytogénétiques. 
Tableau 5: Distance de Mahalanobis entre les classes.

\begin{tabular}{|c|c|c|c|c|c|c|c|c|c|c|c|c|c|}
\hline & Cla1 & Cla2 & Cla3 & Cla4 & Cla5 & Cla6 & Cla7 & Cla8 & Cla9 & Cla10 & Cla11 & Cla12 & Cla13 \\
\hline Clal & 0 & & & & & & & & & & & & \\
\hline $\mathrm{Cla} 2$ & 1.6391 & 0 & & & & & & & & & & & \\
\hline $\mathrm{Cla}_{3}$ & 2.4325 & 3.1886 & 0 & & & & & & & & & & \\
\hline $\mathrm{Cla} 4$ & 2.4794 & 2.3258 & 4.1901 & 0 & & & & & & & & & \\
\hline Cla5 & 2.0631 & 2.4363 & 2.139 & 2.6111 & 0 & & & & & & & & \\
\hline Cla 6 & 2.091 & 2.111 & 3.4539 & 3.4619 & 2.9418 & 0 & & & & & & & \\
\hline $\mathrm{Cla} 7$ & 2.6813 & 2.8292 & 3.4723 & 2.7653 & 2.5957 & 3.2548 & 0 & & & & & & \\
\hline $\mathrm{Cla} 8$ & 3.6478 & 3.8328 & 3.7464 & 4.3166 & 3.4478 & 4.6044 & 3.2405 & 0 & & & & & \\
\hline Clag & 3.8437 & 3.5849 & 4.9442 & 3.8324 & 4.1614 & 3.6822 & 3.8267 & 4.8016 & 0 & & & & \\
\hline Clalo & 2.1112 & 2.8382 & 2.7823 & 3.6743 & 2.9703 & 2.9063 & 2.2468 & 2.9815 & 3.9146 & 0 & & & \\
\hline Clall & 4.0269 & 4.0549 & 4.7845 & 4.6497 & 4.2781 & 3.7448 & 3.5115 & 5.0152 & 4.6344 & 3.5397 & 0 & & \\
\hline Cla12 & 2.4782 & 2.9622 & 3.5102 & 3.8321 & 3.41 & 2.4043 & 2.4758 & 4.2507 & 4.4288 & 2.1652 & 3.6544 & 0 & \\
\hline $\mathrm{Cla} 13$ & 2.3363 & 2.8689 & 3.2012 & 3.9945 & 3.2419 & 2.8658 & 3.7109 & 4.2631 & 4.8152 & 3.0051 & 4.2778 & 2.9203 & 0 \\
\hline
\end{tabular}


R. G. ZANGRE et al. / Int. J. Biol. Chem. Sci. 3(5): 1042-1056, 2009

Tableau 6: Correspondance entre classes agromorphologiques et provenance géographique.

\begin{tabular}{lllcc}
\hline Classe & Localité & Nom local & N écotype & Code \\
\hline Cla1 & $\begin{array}{l}\text { Boucle du } \\
\text { Mouhoun }\end{array}$ & Saya & 28 & bm1 \\
Boucle du & $\begin{array}{l}\text { Mouhoun } \\
\text { Boucle du }\end{array}$ & Yimin & 57 & bm4 \\
Cla1 & $\begin{array}{l}\text { Mouhoun } \\
\text { Boucle du }\end{array}$ & Kazoé & 61 & bm6 \\
Cla1 & Mouhoun & Poundou 2 & 69 & bm9 \\
Cla1 & centre-nord & Kizouiraogo & 1 & $\mathrm{n} 1$ \\
Cla1 & centre-nord & Gomtinini & 5 & $\mathrm{n} 5$ \\
Cla1 & inconnu & & 50 & $\mathrm{in} 2$ \\
Cla1 & nord & Balbou & 21 & $\mathrm{nd} 3$ \\
Cla1 & sahel & Naata1 & 22 & $\mathrm{~h} 7$ \\
Cla1 & sahel & Naata2 & 59 & $\mathrm{~h} 13$ \\
\hline Cla2 & centre-nord & Oudalan & 2 & $\mathrm{n} 2$ \\
Cla2 & centre-nord & Gomtinini & 9 & $\mathrm{n} 9$ \\
Cla2 & sahel & Motiri & 14 & $\mathrm{~h} 2$ \\
Cla2 & sahel & Naata & 17 & $\mathrm{~h} 5$ \\
Cla2 & sahel & Sa dooré & 24 & $\mathrm{~h} 8$ \\
Cla2 & sahel & Sambal & 60 & $\mathrm{~h} 14$ \\
\hline Cla3 & centre-est & Kazoui & 27 & $\mathrm{ce} 1$ \\
Cla3 & centre-nord & Kizouibalkoum2 & 3 & $\mathrm{n} 3$ \\
& & & &
\end{tabular}

\begin{tabular}{|c|c|c|c|c|}
\hline Classe & Localité & Nom local & $\mathbf{N}$ écotype & Code \\
\hline Cla7 & centre-est & & 29 & ce2 \\
\hline Cla7 & centre-est & Kii & 66 & ce3 \\
\hline Cla7 & $\begin{array}{l}\text { centre-sud } \\
\text { centre-sud }\end{array}$ & Yirsam & 23 & $\mathrm{c} 1$ \\
\hline Cla7 & & Ki poolga & 31 & $\mathrm{c} 4$ \\
\hline Cla7 & centre-sud & Kignanga & 47 & c9 \\
\hline Cla7 & centre-sud & Ki-raaga & 56 & $\mathrm{c} 12$ \\
\hline Cla7 & centre-sud & Ka-yaaga & 62 & $\mathrm{c} 13$ \\
\hline Cla7 & sud & Sorgo & 36 & s5 \\
\hline Cla8 & centre-sud & Yirda & 26 & $\mathrm{c} 2$ \\
\hline Cla8 & sud & Kiraaga & 41 & $\mathrm{~s} 10$ \\
\hline Cla9 & Boucle du Mouhoun & Poundou 1 & 68 & bm8 \\
\hline Cla9 & ouest & & 67 & ou1 \\
\hline Cla9 & sahel & Naadari & 30 & h10 \\
\hline Cla9 & sahel & Gawri udaala & 65 & h15 \\
\hline Cla10 & centre-sud & Ka pelga2 & 32 & $c 5$ \\
\hline Cla10 & centre-sud & Kapelga1 & 45 & c7 \\
\hline Cla10 & centre-sud & Kignanga & 46 & $\mathrm{c} 8$ \\
\hline Cla10 & centre-sud & Kirimangué & 51 & $\mathrm{c} 11$ \\
\hline 1052 & & & & \\
\hline
\end{tabular}


R. G. ZANGRE et al. / Int. J. Biol. Chem. Sci. 3(5): 1042-1056, 2009

\begin{tabular}{|c|c|c|c|c|}
\hline Cla3 & centre-nord & Kizouibalkoum1 & 4 & $\mathrm{n} 4$ \\
\hline Cla4 & centre-nord & Kazouizuwooko & 6 & n6 \\
\hline Cla4 & centre-nord & $\begin{array}{l}\text { Kazuizunodo } \\
\text { pegpedo }\end{array}$ & 10 & $\mathrm{n} 10$ \\
\hline Cla4 & sahel & Naata & 53 & h11 \\
\hline Cla5 & $\begin{array}{l}\text { Boucle du } \\
\text { Mouhoun }\end{array}$ & Mondri & 58 & bm5 \\
\hline Cla5 & centre-nord & Bagkièma & 7 & $\mathrm{n} 7$ \\
\hline Cla5 & centre-nord & Kazouizowooko & 8 & n8 \\
\hline Cla5 & centre-nord & Kazouisagkioma & 12 & $\mathrm{n} 11$ \\
\hline Cla5 & nord & Balbo & 18 & nd1 \\
\hline Cla5 & nord & Kakouèga & 20 & nd2 \\
\hline Cla5 & sahel & Balma & 19 & h6 \\
\hline Cla6 & $\begin{array}{l}\text { Boucle du } \\
\text { Mouhoun } \\
\text { Boucle du }\end{array}$ & Sayon1 & 54 & bm3 \\
\hline Cla6 & Mouhoun & Sayon2 & 63 & bm7 \\
\hline Cla6 & sahel & Sambalé & 13 & h1 \\
\hline Cla6 & sahel & Kazoui2 & 15 & h3 \\
\hline Cla6 & sahel & Kazoui1 & 16 & $\mathrm{~h} 4$ \\
\hline Cla6 & sahel & Sambal & 25 & h9 \\
\hline Cla6 & sud & Adoré & 11 & s1 \\
\hline
\end{tabular}

\begin{tabular}{lllcc} 
Cla10 & centre-sud & Yir-ya & 64 & c14 \\
Cla10 & sud & Kignanga1 & 35 & s4 \\
& sud & Kignanga2 & 39 & s8 \\
Cla10 & sud & Kapelga & 43 & s12 \\
Cla10 & Kignanga4 & 33 & s2 \\
Cla11 & sud & Kignanga5 & 34 & s3 \\
Cla11 & sud & Kipelga & 44 & $\mathrm{c} 6$ \\
\hline Cla12 & centre-sud & Kignanga3 & 48 & $\mathrm{c} 10$ \\
Cla12 & centre-sud & Sorgo2 & 37 & $\mathrm{~s} 6$ \\
Cla12 & sud & Sorgo1 & 38 & $\mathrm{~s} 7$ \\
Cla12 & sud & Kipelga & 40 & $\mathrm{~s} 9$ \\
Cla12 & sud & Kignanga & 42 & $\mathrm{~s} 11$ \\
\hline Cla12 & sud & Yimin & 52 & $\mathrm{bm} 2$ \\
\hline \multirow{2}{*}{ Cla13 } & Boucle du Mouhoun & & 49 & in1 \\
Cla13 & inconnu & Garel-dabbel & 55 & $\mathrm{~h} 12$ \\
\hline Cla13 & sahel & & &
\end{tabular}




\section{Stratification de la variabilité de la collection}

La variabilité de la collection a été décrite grâce à l'analyse en composantes principales suivie de la classification ascendante hiérarchique et l'analyse factorielle discriminante qui mettent en exergue quinze (15) variables permettant le plus de distinction entre les écotypes et sont pour ce fait retenues comme variables actives tandis que les autres sont pris comme variables supplémentaires.

Les caractères tels que l'intervalle semi-épiaison, la hauteur à maturité, le nombre des talles basales, la morphologie de l'épi et de la feuille étendard offrent une meilleure différenciation phénotypique des écotypes du mil ; confirmant ainsi les travaux de Balma (1992).

La classification ascendante suivie de la troncature automatique de l'arbre hiérarchique a permis la constitution de 13 classes d'individus semblables exhibant $88 \%$ de variation interclasse. L'analyse discriminante donne $78 \%$ de reclassement, ce qui confirme le bien fondé de ces classes.

La structure de la population décrite par la troncature successive de l'arbre hiérarchique montre que la première troncature qui est expliquée à $97 \%$ par le plan 1-2 scinde la population en deux groupes: d'une part les écotypes précoces, de petite taille, possédant des épis longs et des rendements élevés et d'autre part des écotypes à cycle long, de grande taille avec des épis courts et un rendement faible. Ces résultats rejoignent ceux de Zangré (1979) et de Zangré et al. (1992) qui distingue deux grands groupes de mil au Burkina Faso. Le premier groupe correspond aux écotypes du nord, du centre-nord, du sahel, de la boucle du Mouhoun et du Houet, tandis que le second regroupe ceux du sud, du centre-sud et du centre-est. Ces deux groupes montrent des différences hautement significatives pour la plupart des variables à l'exception de la largeur de l'épi, la largeur de la tige, le nombre d'épis récoltés, le rapport grains sur épi et le nombre d'épis par plante. On retrouve cependant un écotype du sud et un du centreest dans le premier groupe. En effet il s'agit de l'écotype précoce Iniadi originaire du sud Burkina-nord Ghana qui se retrouve avec les écotypes du nord á cause de sa précocité. Tout laisse croire que la première scission de la population est beaucoup basée sur le cycle de la plante. Cette structuration rejoint celle proposée par Anand Kumar et Appa Rao (1987) qui voient un gradient de précocité du nord au sud sans que les écotypes précoces soient exclusivement confinés dans le nord mais aussi cultivés au sud. La structuration de la population sur la base des caractères agromorphologiques a entraîné du même coup une structuration régionale. La seconde troncature ainsi que les autres ont contribué à segmenter la population en des groupes agromorphologiques de plus en plus homogènes mais n'ont pas révélé une structuration intra-région. Toutefois on remarque une position excentrée de l'écotype du Houet mais la faiblesse de l'échantillon ne permet pas de tirer des conclusions adéquates.

Un croisement qui réunirait des éléments des classes les plus éloignées favoriserait un maximum d'hétérosis. Les classes 3, 4, 5 et 8 renfermant 15 écotypes sont les plus performantes en termes de rendement. D'autres classes peuvent être retenues pour des performances diverses : la classe 9 pour la longueur de l'épi, la longueur et la largeur du drapeau ainsi que le poids de l'épi et la classe 3 pour le tallage.

La concordance entre structuration agromorphologique et structuration géographique montre l'importance du facteur régional dans la répartition de la diversité génétique. Cette étude mérite d'être plus poussée afin de définir des unités d'aires géographiques de diversité génétique nécessaires pour une meilleure collecte et une 
meilleure conservation in situ des ressources phytogénétiques du mil.

\section{Conclusion}

Notre étude a montré que la collecte des écotypes locaux avec la participation des paysans détenteurs et utilisateurs du mil permet de constituer une collection d'une grande variabilité génétique.

Les analyses multivariées basées sur les caractères agromorphologiques, le cycle et le rendement, permettent réellement une structuration de la variabilité génétique. Lorsque la structuration est faite sur la base du cycle et des caractères morphologiques, elle coïncide avec la structuration géographique des écotypes. Le cycle de la plante, la longueur de l'épi, la hauteur de la plante et le structuration de la population. Les paysans du Nord sélectionnent pour des écotypes à cycle diamètre du pédoncule sont les caractères qui ont contribué le plus à la court de moins de 90 jours tandis que ceux du Sud sélectionnent pour des écotypes à cycle long mais aussi pour des écotypes à cycle court. En plus du caractère cycle de la plante, les écotypes du Nord se distinguent de ceux du Sud par plusieurs traits morphologiques. Lorsque la distinction entre écotypes est faite en incluant le rendement, les limites entre écotypes du Nord et écotypes du Sud ne sont pas distinctes. Les paysans de ces deux régions sélectionnent donc tous pour la productivité. Il apparaît alors clairement que les critères de sélection du paysan ont influencé la variabilité génétique. Une bonne connaissance des objectifs et des critères de sélection du paysan pourrait contribuer à la conception de stratégies efficaces de conservation in situ de la variabilité génétique.

D'autre part les caractères présentant de fortes interactions génotype-environnement sont défavorisés par l'évaluation dans le seul site de Gampéla. IL y'a donc une nécessité d'effectuer des évaluations pluriannuelles et multilocales afin de mieux structurer la variabilité génétique. Elle permettra d'identifier des régions de grande diversité génétique où on pourra effectuer les collectes et la conservation in situ des ressources phytogénétiques.

\section{REFERENCES BIBLIOGRAPHIQUES}

Anand Kumar K, Appa Rao S. 1987. Diversity and utilisation of pearl millet germplasm.. In Proceedings of international pearl millet workshop, Witcombe JR, Beckerman SR (eds). ICRISAT: Patancheru, India; 69-82.

Balma D, 1992. Etude de la variabilité génétique du mil (Pennisetum typhoides Burm. Stapf et Hubb.)-Implications concernant la conservation et l'utilisation des ressources phytogénétiques. Thèse du grade de Ph.D. Faculté des Sciences de l'Agriculture et de l'Alimentation Université Laval (Québec) Canada, p.188.

Beninga M. 1992. Evaluation et utilisation des ressources génétiques des mils et sorgho. Collecte et valorisation des formes sauvages. In Complexes d'espèces, flux de gènes et ressources génétiques des plantes. Colloque international du 8-10 janvier 1992 en hommage á Jean Pernes, Professeur á l'Université d'Orsay, Paris XI, Paris. Bureau des Ressources Génétiques, 57, rue Cuvier, F-75231 Paris cedex 05, p. 73-86.

Christinck A, vom Brocke K, Kshirsagar KG, Weltzien E, Bramel-Cox PJ. 2000. Participatory methods for collecting germplasm: Experiences with farmers in Rajasthan, India. Bulletin de Ressources Phytogénétiques, IPGRI-FAO, 121: 2026.

Frankel OH, Brown AHD. 1984. Plant genetic resources today: A critical appraisal. In Crop Genetics Resources: Conservation and Evaluation, Holden JHW, Williams JT (eds). George Allen and Unwin: London, UK; 249-257.

Malosetti M, Abadie T, Germán S. 2000. Comparing strategies for selecting a core 
subset for the Uruguayan barley collection. Bulletin de Ressources Phytogénétiques, IPGRI-FAO, 121: 2026.

Obilana AB, Manyasa E. 2002. Millets. In Pseudocereals and Less Common Cereals: Grain Properties and Utilization Potential, Belton PS, Taylor JRN (eds). Springer-Verlag: New York; 177-217.

Pernes JD, Combes, Leblanc JM. 1984. Le mil. In Gestions des Ressources Génétiques des Plantes. Tome 1 : Monographies, Pernes J (ed). Agence de Coopération Culturelle et Technique: Paris, France ; 159-210.

Rai KN, Murty DS, Andrews DJ, Bramel-Cox PJ. (1999). Genetic enhancement of pearl millet and sorghum for the semi-arid tropics of Asia and Africa. Genome 42: 617-628.

Zangré GR. 1979. Recherche de critères de sélection pour la tolérance à la sécheresse chez le Mil. Mémoire de fin d'études de D. A. A. ENSA, p.103.

Zangré, R., Nguyen-Van, E., Rherissi, B., and Till-Bottraud, I. 1992. Organisation du pool génique de Setaria italica (L.) P.Beauv. et exploitation des ressources génétiques d'espèces spontanées. In Complexes d'Espèces, Flux de Gènes et Ressources Génétiques des Plantes. Lavoisier édition BRG : Paris ; 87-97. 